\title{
Card Sorting Activities with Preschool Children
}

\author{
Ana Vitoria Joly \\ University of Brighton
}

\author{
Lyn Pemberton \\ University of Brighton \\ Lewes Road, BN2 4GJ \\ Brighton, United Kingdom
}

\author{
Richard Griffiths \\ University of Brighton
}

\author{
+44 (0) 1273642476 \\ A.V.B.Joly@brighton.ac.uk Lyn.Pemberton@brighton.ac.uk R.N.Griffiths@brighton.ac.uk
}

\begin{abstract}
Preschoolers' categorization differs from adults'; therefore it is beneficial to involve them in the design process to create appropriate information architecture for this age group. In this paper, we describe three card sorting activities conducted with a total of fifty six preschool children in five nurseries. We conclude that a closed card sorting task can be combined with a matchto-sample activity to offer insight into children's categorization knowledge, contributing significantly to the design of technology for young children.

\section{Keywords}

Card sorting, preschool children, user-centred design

\section{INTRODUCTION}

Card sorting techniques have been used to create information architecture eliciting conceptual structures from participants in order to reflect how users view the content [11]. In a conventional card sort activity participants are asked to sort a group of cards with concepts written on them, usually menu entries, categories or headings. After the cards are sorted they are asked to label each group and explain the sorting criteria used. Researchers then study the number of sorts, groups within sorts, the similarity in groups, and the sense of distance of concepts grouped together [7].

In open card sorts, recommended for exploration, the number and names of groups are decided by each participant, while in closed card sorts, suggested for assessment, these factors are fixed by the researcher in advance [5].

Card sorting tasks have been carried out with children as young as eight years of age [4]. In this paper we discuss the way this technique can be adapted to be used with 3 and 4 year old children and contribute to the design of technology for this age group.

This study is part of a larger research on preschoolers' interactions with interactive television, in which these card sort activities were used to define and refine categories for an Electronic Programme Guide (EPG) to assist children to find videos using the remote control and

(C) The Author 2009.

Published by the British Computer Society the mouse. Three different studies are reported along with advantages and limitations of both open and closed card sort activities.

\section{CHILDREN'S CATEGORICAL KNOWLEDGE}

According to Murray and Reuter (2005), research into children's acquisition of categories helps us understand the impediments to children's use of traditional classification schemes [9]. Attention to this literature offers an understanding of children's cognitive and developmental needs, which should influence the design of classification schemes and information retrieval systems to better accommodate children's information needs and abilities.

A hundred years of research in developmental psychology suggests that preschool children often categorize by common perceptual properties and/or by thematic relationships [13]. Goswami (1998) supports the view that child-basic categories differ from adult-basic categories since children may notice or emphasize different attributes of the same object than adults. Because of their different experiences and their different knowledge, children may then give priority to characteristics rather than defining features in making their category judgments [3]. As a result, categories established by adults may not reflect children's classification; for that reason, in order to create userappropriate information architecture, is necessary to involve children in the design process.

\section{CLOSED CARD SORTING}

Several categorization activities in psychology studies are based on a task in which children are given a target object and are asked to select the appropriate match from a pair of alternatives. So we decided to design the first card sorting activity using triads as well, inspired by the Dimensional Change Card Sorting (DCCS) task used to determine extradimensional shifting abilities in preschool children [6]. For the EPG prototype under development, an initial set of categories were pre-established based on children's TV channels' websites, children's film and book categories. This resulted in nine categories: "Movies", "Cartoons", "Music", "Make and Do", "Animals and Nature", "Fairy Tales", "Superheroes", "Around the World" and "TV Shows" (including live action and puppet shows). We also decided to test whether children associate video content with the channel that broadcasts it, if so channels would also be categories. As a result in addition to sorting a member of each one of the nine categories we also asked children to sort two 


\section{A. Joly et al.}

screenshots members of two different channels, "CBeebies" and "Disney", totalling eleven screenshots to be sorted. The objective of this study was to check how well these pre-established categories fit children's expectations.

In the Dimensional Change Card Sort (the inspiration for this activity) children are asked to place cards into trays according to simple rules such as colour and shape. The card sorting experiment described in this paper, however, requires more abstract categorization ability, involving blurred categories, such as "Cartoons" and "Movies", that sometimes merge and imposing participants' understanding that the screenshots presented are representations of video content. Therefore, before confirming if the pre-established categories were appropriate the card sort activity needed to be validated and give evidence that preschoolers can categorize videos based on still screenshots.

\section{Method}

\section{Participants}

The sample size needed to validate the closed card sorting activity was defined with the assistance of a statistician using the tables for logistic regression and resulted in fifty to sixty children.

Letters were sent to all nurseries in Brighton (UK) explaining the study, one week later the nurseries were contacted by phone, some of them declined to participate, in others, managers could not be reached on the first attempt. But five of them agreed to take part in the study providing the sample needed.

The inclusion/exclusion criteria used were children's age group and a signed consent form. Children who took part in the study were 3 and 4 years old, whose parents had signed a consent form allowing them to participate and who wanted to take part after the research was briefly explained to them.

Before the data collection started, the research project was approved by the University Ethics Committee. To protect children's privacy, all data collected was anonymised and confidentiality maintained. Children were referred to by their first name during the activities, but in the data collected their names were replaced by a participant number and the data analysis included only these numbers.

There were six sessions conducted in five different nurseries. The activity was carried out in a corner of the Nursery setting or in a separate room under the supervision of a member of Nursery staff. Fifty six participants contributed to the study, twenty seven girls and twenty nine boys, aged between 36 and 56 months, with a mean age 46 months. Most children were from white middle-class backgrounds, but this data was not systematically collected.

Four additional children were excluded from the study; two who inserted all cards in the same box and did not show sufficient understanding of the task, and two due to researcher's error.

\section{Materials}

Two side-by-side shoeboxes (Figure 1) were designed, each with a plastic sheet to display a pre-established category plus a slot through which the participant should post a $9 \mathrm{~cm} \times 6 \mathrm{~cm}$ laminated card showing a screenshot from a video. Two predefined categories were displayed at a time and the child's task was to post the screenshot in the box they found more appropriate. For the complete list of the triads, screenshots and the two choices of categories presented, see Appendix A.

There were icons representing each category but the names of the categories were read out for clarity. These icons were chosen with children during previous design sessions.

Some of the screenshots presented were part of a video content while others were from promotion material, such as the one from the movie Ratatouille on Figure 1. The videos chosen were children's programmes currently being broadcast and popular children's films and were all strong members of one of the categories presented. The screenshots selected represented the essence of the corresponding video content.

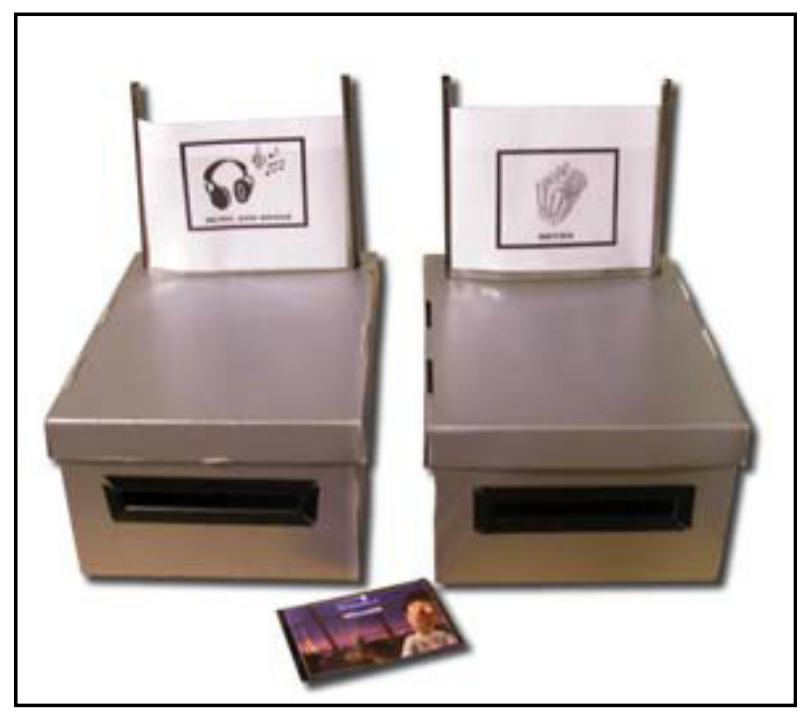

Figure 1. Closed card sorting set up.

The triads composed of one screenshot and two categories were kept the same, but participants were randomly assigned to one of three different conditions, each condition with a random order of triads. This randomization was found necessary because in a pilot test most children were mistaken on the three last triads, which could be due to their tiredness at the end of the activity.

The screenshots were printed in colour and the category icons were black and white, to avoid colour associations.

\section{Procedure}

Each session lasted approximately ten minutes and the task was carried out individually by each child.

First the child was asked if $\mathrm{s} / \mathrm{he}$ watched television and what was his/her favourite programme. Then it was explained his/hers help was needed to develop a TV guide for children. The child was asked if $\mathrm{s} /$ he could help and it was mentioned that if $\mathrm{s} / \mathrm{he}$ decided not to help or to 
stop helping at anytime it was acceptable. The card sorting activity was then described.

The screenshot was shown and it was asked if the child recognized it. If $\mathrm{s} /$ he did, the child was asked to post it into one of the two boxes. If the child did not recognize the screenshot, another one of the same category was shown. If the child did not recognize the second screenshot shown, s/he was asked to choose one between the two screenshots of that category and place it in the more appropriate box. Two new categories would then be displayed. In all, eleven screenshots would have been sorted.

The first and main objectives of the task were to check if children were able to relate the screenshot to the video and then categorize the video content. For this reason it would be important that participants were familiar with the video so they could recognize it from the screenshot and categorize it. Hence it was decided to provide two options instead of one to increase the chances participants were acquainted with the video. The second screenshot was usually part of a video that was also a strong member of the same category that the first screenshot shown belonged but from a different channel, broadcast at a different time of the day, or mainly directed to a different gender. In case a child did not recognize any of the two screenshots presented we decided to ask her/him to sort one of them anyway in order to verify if a screenshot could contain enough information to support children's categorization of a video.

After each screenshot had been sorted the researcher said "Thanks" but no positive or negative feedback was given. When children finished sorting the eleventh screenshot they received a certificate to thank them for their participation.

\section{Results}

The closed card sorting activity was a binomial experiment in which each trial could result in only one of two outcomes, success in case the child posted the screenshot in the expected box (category which the screenshot was a strong member of, according to adult classification) or failure if the child posted the screenshot in the unexpected box or refused to post it in any box. Success and failure are related to the pre-established categories, in case a significant number of children post a screenshot in the unexpected box this would indicate a failure of the category itself, and that it should probably be modified or eliminated. We have also used the term "accuracy" to refer to the degree of correspondence to the pre-established categorization.

Three participants refused to categorize specific screenshots because they were unsure to which category it could belong. Two participants refused to categorize the screenshot from the "Animals and Nature" category and one participant refused to categorize five out of the eleven screenshots.

The binomial distribution was used to validate the activity and calculate if the results from the closed card sorting task were above chance. The null hypothesis tested was that the sample data do come from a B (11, 0.5) distribution, implying that the participants are guessing. A probability value (P-Value) cut-off of 0.05 was then used to reject this hypothesis in favour of an alternative hypothesis that the sample data do not come from a B (11, 0.5) distribution, which imply that participants are able to categorize video content using screenshots and the card sort activity is valid. Since the observed and expected values were very different and the resulting P-Value less than 0.0005, the null hypothesis was rejected. There is very strong evidence that participants were not guessing and the closed card sorting task can be conducted with preschoolers.

Correlation was used to measure the relationship between the number of screenshots that were posted in the expected category and participants' age. Deak et al. (2002) concluded in their study that children's inference making and similarity selection, such as sorting, are best regarded as "phenomena that emerge under constellations of properties of the child and his/her environment" [1]. According to Deak et al. preschoolers' matching performance depend on several factors and is difficult to accommodate under traditional theories of cognitive development such as Piaget that emphasizes age-related changes in static representational structures or capacities.

In this study, from the scatterplot produced (Figure 2), it can be noticed that there is no relationship between the number of screenshots posted in the expected box and child's age. So, within this age group, there isn't any indication that as participants get older they will form categories more similar to adults'. This could mean that other factors also interfere in the categorization ability. Although every participant affirmed s/he watches television, the frequency of media use and variety of content watched, for instance, could have more influence on how preschoolers categorize videos than the child's age.

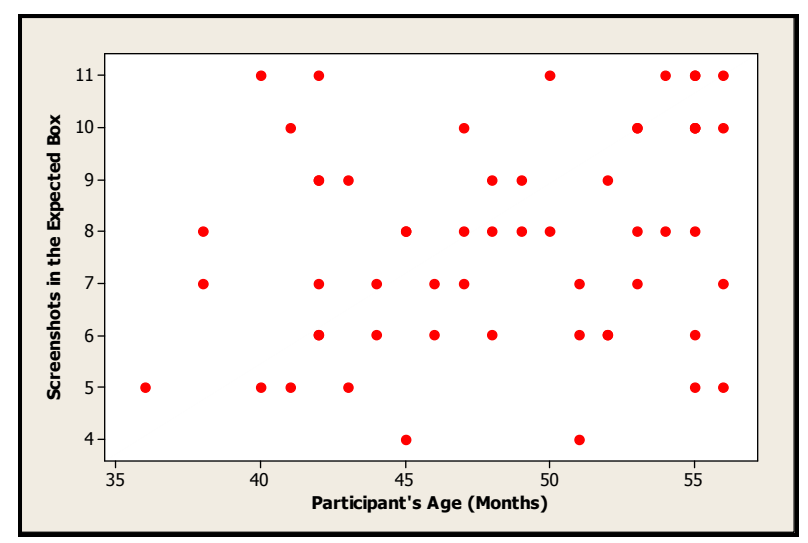

Figure 2. Scatterplot of the number of screenshots posted in the expected box versus participant's age in months.

The Mann-Whitney test suggests that there is no significant difference between the mean accuracy for boys and girls. Therefore we can conclude that there is not a significant relationship between children's categorization ability and their gender.

A Kruskal-Wallis test indicates that the median accuracy is very similar for the three conditions. Thus, the condition did not significantly affect the results.

Another Kruskal-Wallis test was conducted to verify the impact the Nursery could have on the median accuracy 
and it indicated that there is a difference in the medians. As it may be seen on the boxplot below (Figure 3) the median accuracy in Nursery 1 appears to be higher than those in Nurseries 2, 3, 4 and 5.

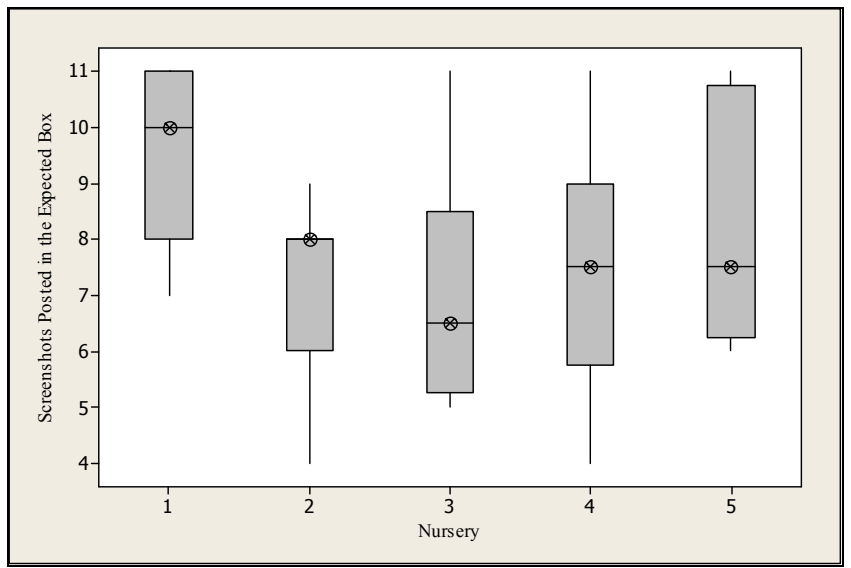

Figure 3. Boxplot of the number of screenshots posted in the expected box versus each Nursery.

This difference could have occurred because the session in Nursery 1 was the only one conducted in a quiet separate room while the sessions in the other nurseries were conducted in a corner of the Nursery setting. The environment noise and activity could have interfered with participants' concentration and influenced the results.

Most participants instantly recognized the first or second screenshots shown and related it to the video it represented. The only exception was the screenshots from the category "Animals and Nature". None of the participants recognized any of the videos from either of the two screenshots presented. This was probably because the videos the screenshots came from were not broadcast regularly and not very popular. Nevertheless, despite not being familiar with the video, a significant number of participants posted the "Animals and Nature" screenshots into the expected box (see Table 1). This indicates that a screenshot can contain enough information for children to be able to categorize its video content.

Given that the results from the closed card sorting task provide evidence that 3 and 4 year old children are able to relate a screenshot to the video it represents and categorize it. This activity can be used to assist on the design of technology for young children.

In this research the card sorting task was used to define categories for a children's EPG. The results indicate which categories are better understood and which ones may not be as comprehensible for preschoolers.

The numbers were calculated according with the preestablished categories, success as 1, matching the expected categorization, and failure as 0 , when children chose the unexpected category or refused to categorize. The mean accuracy for each pre-established category presented in the table below was calculated to support design decisions.

\begin{tabular}{ll}
\hline Categories & $\begin{array}{l}\text { Mean Accuracy } \\
\text { (Standard Deviations) }\end{array}$ \\
\hline Movies & $0.77(0.426)$ \\
Cartoons & $0.68(0.471)$ \\
Music & $0.63(0.489)$ \\
Make and Do & $0.54(0.503)$ \\
Animals and Nature & $0.73(0.447)$ \\
Fairy Tales & $0.77(0.426)$ \\
Superheroes & $0.89(0.312)$ \\
Around the World & $0.59(0.496)$ \\
TV Shows & $0.61(0.493)$ \\
CBeebies (channel) & $0.80(0.401)$ \\
Disney (channel) & $0.84(0.371)$ \\
\hline Table 1. Mean Accuracy (Standard Deviations) of \\
Categorizing Screenshots into Pre-Established \\
Categories.
\end{tabular}

The Table 1 suggests that some categories are understood better than others. Given that $89 \%$ of the participants posted the superheroes screenshots into the expected box labelled "Superheroes", we can affirm that this category is understood by most preschoolers.

Categories like "Make and Do" and "Around the World" seemed to be particularly difficult for children. Considering $\mathrm{p}=0.5(50 \%$ chance of success and $50 \%$ chance of failure), those two categories are just above chance. For this reason they need to be refined or eliminated from the EPG.

Despite the increasing number of children's channels available broadcasting the same content and the video on demand features that can disconnect the content from the channel, it was significant the number of children who recognized the channel in which the correspondent video content is broadcasted. As a result children's channels will be included as categories in the prototype.

The main design decisions resulted from this activity were: "Animals and Nature" should be maintained as a category in the EPG, despite the screenshots were not recognized, because a significant number of children would identify the category and be aware of the type of its content. The "Make and Do" and "Around the World" categories should be refined or eliminated. Children's channels should be included as categories in the EPG.

\section{OPEN CARD SORTING}

The categories tested by the closed card sorting were made for children, by adults. There was a concern that child based categories of video content could be completely different from what was pre-established based on existent categorization, so we decided to experiment other card sorting activities to complement the closed card sorting task.

On the hierarchical taxonomic concept test [14] children were presented with four index cards and two baskets and asked to put cards in different baskets according to their 
categories. After the classification was made a further four index cards would be presented, with two more baskets and children asked to explain the categorization. Initially, our intention was to provide boxes to replicate this study with the screenshots. However, we decided to ask children to make piles instead and group the screenshots together, otherwise the number of categories that could emerge from the activity would be limited.

\section{Method}

\section{Participants}

This activity was conducted in one Nursery in Brighton (UK). The inclusion criteria were children's age and a signed consent form.

After the closed card sorting task, children were asked if they wanted to continue to help the researcher by playing another game. Eight participants, seven girls and one boy, aged between 40 and 55 months, mean age 47.6 months, decided to carry out the open card sorting.

\section{Materials}

Twenty $9 \mathrm{~cm}$ x $6 \mathrm{~cm}$ laminated cards showing a screenshot from a video were used.

There were two different conditions. In condition 1 , children were given five sets of four screenshots each and in condition 2 children were given four sets of five screenshots each. For a complete list of screenshots given in each set for each condition see Appendix B.

\section{Procedure}

The activity was conducted individually with each participant in a session that lasted for approximately ten minutes.

The activity was briefly explained to the participants as the grouping game, in which they had to put together things that are the same type or kind. The first three participants were assigned to condition 1 while the five last participants were assigned to condition 2. Children were given some time to make groups before the next set was presented. The screenshots in each of the sets were shuffled before being handed to the participant.

Children were motivated to consider all screenshots while making groups, not just the separated sets. And at the end of the activity children were asked to justify their choices.

\section{Results}

The three first participants put together two different sets of two screenshots, strong members of the same category, from the first set but then struggled to continue the activity when the number of screenshots increased. For this reason the five following participants, were assigned to condition 2, in which it was given four instead of five sets and distributed these members of the same category that were being easily sorted to check if this was due to their similarity or because they were both on the first set.

All participants gathered only two screenshots in each group they have made. Most of the screenshots put together were strong members of a category and/or had strong perceptual similarities.

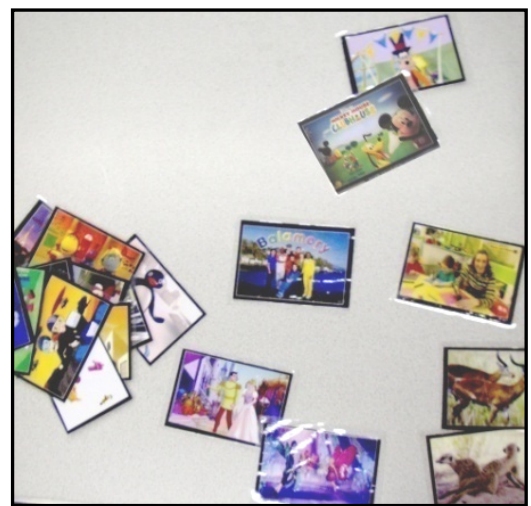

Figure 4. Cards Sorted by a Participant

Children were asked about their decisions but they could not explain or justify their choices.

There were two screenshots from the same video and although it contained different characters in different scenarios, seven out of eight participants grouped them together. This reinforces the finding that preschoolers are able to relate the screenshot to the video content it represents.

Several groups were formed by members of the preestablished categories. Seven children gathered the two screenshots from the category "Animals and Nature". Six children put the two screenshots representing "Fairy Tales" in the same group. Three children gathered the two screenshots from videos broadcasted in the same channel. And two children made a group with the two screenshots containing images from music videos, that would be part of the pre-establish category "Music".

But non-expected groups were also formed, most of them containing cartoons and animations, including 3D and stop motion. In the pre-established categories of the EPG the category "Cartoons" excluded animations. Animations were categorized as "TV Shows" if they were TV programmes or "Movies" if they were from films. However, from the open card sorting activity we noticed that children do not differentiate cartoons, 3D animations and stop motion animations.

This activity has to be further developed to help in the design. Children usually become overwhelmed when too many options are shown and cannot associate them, nor can they explain their choices. It may require more of participant's time and would be probably necessary to have several sessions with children to achieve some level of contribution to the information architecture of a system. As a result, the open card sorting could be an interesting technique to be used when young children are technology design partners and contribute to the design process throughout the experience [2].

In this research however children are informants [12], they are asked for input at some stages of the design process and play some part in informing the design but their participation is limited as well as the time they collaborate. Hence we found necessary to find another activity to complement the closed card sorting that would not be as time consuming as the open card sorting. 


\section{MATCH-TO-SAMPLE}

The results from the closed card sorting indicate which categories are well understood by children and which ones are not as clear. In case most participants relate a screenshot to the expected category the design decision is simple, to maintain that category. However when a category is not comprehended the design decision could be either eliminate or refine the category, and to do so it is essential to identify to which other category children would relate its members. For example, if most children did not insert the make and do screenshot into the expected "Make and Do" category box, it demonstrates that the category is not well understood, but it does not indicate to which other category the make and do screenshot should belong.

In order to confirm the closed card sorting results and find if the screenshots that were not inserted into the expected category could fit within another preestablished category instead we developed the match-tosample activity.

The initial idea for the activity was to ask participants to choose a category between the eleven pre-established ones to paste a screenshot. Nevertheless, this could offer too many options and could overwhelm children like the open card sorting task. So we decided to base the task on an existent activity to measure children's categorization ability.

Mervis and Pani (1980) used twenty four objects forming six artificial categories with four members each. In their experiment 5 year old children were taught the name of six objects, one in each category. They were then presented with all objects; the researcher would say the name of one object and asked the participant to point or touch all the objects $\mathrm{s} /$ he would call by that name. The same procedure was repeated for each of the six category names [8].

We adapted this study organizing twenty four screenshots into six different groups of four screenshots each. The groups were made by some of the pre-established categories and the screenshots to be sorted were some of the ones used in the closed card sorting.

During a pilot study we tested the categories "Cartoons", "Make and Do", "Superheroes", "TV Shows", "Movies" and "Fairy Tales". In the pilot test there was only one condition in which children had to paste one screenshot for each one of the six categories. We found however, probably due to the fact that this was the second activity conducted with each child, participants looked tired after pasting the three first screenshots. So we decided to ask each child to paste only three screenshots each and assigned participants to one of three different conditions.

Also in the pilot test we confirmed the open card sorting result that most children did not differentiate cartoons from other types of animations. Most children pasted the cartoon screenshot with the TV shows' screenshots that included animations. As a result, we decided to test a modified version of the "Cartoons" category labelled "Cartoons and Animations", with one screenshot from a cartoon in condition 1 and one from a 3D animation in condition 3. In addition to that we tested the categories "Movies", "Superheroes", "TV Shows", "Fairy Tales", "Make and Do", "Music" and "TV Shows".

Some of the pre-established categories were left out of the match-to-sample. The categories related to children's channels were eliminated from this task because they seemed to be clear enough. It was the only labelling children did without being requested. During the closed card sorting a significant amount of children related the screenshots to the channel where the video is broadcasted, not only while sorting screenshots from channels categories, but when any screenshot was shown several participants instantly identified the channel the videos were from without being asked to do so.

The "Animals and Nature" category was not included in this activity due to the fact that the screenshots were not recognized by any participant during the closed card sorting, so children would probably not relate the screenshot to the video in the process of categorization as it would be preferable.

The "Around the World" category was also excluded because during the closed card sorting just a few children related the screenshots to the videos and the percentage of participants who associated the around the world screenshots to the expected category was low.

On the other hand, most participants recognized the videos from the make and do screenshots. For this reason, despite the fact that a low percentage of participants related the make and do screenshots to the "Make and Do" category in the closed card sorting, we decided to test this category in the match-to-sample activity to check if the category should be redefined and/or relabelled or if it should be eliminated.

In the match-to-sample task participants were given a screenshot that was a strong member of one of the preestablished categories, but instead of choosing the category it should belong to, they had to choose the group of other similar screenshots that would be exemplars of its category. We believed that the fact that children would not categorize using a category label and icon relating to it, but would be able to choose a group of strong members of the same category would offer a different approach from the closed card sorting that could complement the activity.

\section{Method}

\section{Participants}

This activity was conducted in two nurseries in Brighton (UK), following the closed card sorting session. Like the previous activities, the inclusion criteria were children's age and a signed consent form.

Following the closed card sorting activity, children were asked if they wanted to do another activity. Fifteen children, eight girls and seven boys, aged between 40 and 56 months, with the mean age 49.8 months, participated in the match-to-sample task.

\section{Materials}

We used three A4 sheets on which were printed in colour twenty four screenshots, measuring $3 \mathrm{~cm} \times 2 \mathrm{~cm}$ each, 
organized into six columns of four screenshots each. Each column was composed of strong members of the same category, based on the pre-established categories. Three stickers, also measuring $3 \mathrm{~cm} \times 2 \mathrm{~cm}$, in which was printed in black and white, to avoid colour association, the screenshots to be matched, one sticker to be pasted in each A4 sheet.

Children were randomly assigned to one of three different conditions in which they were given three screenshots, one at a time, and were presented with six different categories to match the screenshot. For the complete list of categories and screenshots for each condition see Appendix C.

\section{Procedure}

Each child carried out the activity individually in a session that lasted for approximately five minutes.

Children were given a sticker with a black and white screenshot and asked to put it on the group they thought it would be most appropriate. After the child had chosen a group and had pasted the sticker, s/he was given another A4 sheet with similar categories, but in random order and composed of different screenshots, and another screenshot to be pasted. No feedback was given during the task, but when children were stuck or asked for help the pre-established category of the screenshot to be matched was labelled. If a screenshot from the "Fairy Tales" category was to be sorted, for instance, in case children needed help the researcher would say the screenshot was from a fairy tale video and would ask the participant to paste it where s/he thought are other fairy tales.

After the three different screenshots were pasted on three A4 sheets children were thanked for their participation.

\section{Results}

The match-to-sample activity gave some insight on how children would fit screenshots into categories already formed by other screenshots.

As expected from the closed and open card sorting activities children did paste the screenshots from a cartoon and a 3D animation in the "Cartoons and Animations" category. The sample was too small to generate results that are statically relevant, but four out of nine participants that were assigned to a condition in which they were asked to paste a screenshot from a cartoon or animation included it with the other members from the "Cartoons and Animations" category, whilst the probability to do so was $1 / 6, p=0.17$. This suggests that children may associate both cartoons and animations to the same group.

The make and do screenshot was not pasted within members of the "Make and Do" category by any child. Four out of six participants who were asked to paste this screenshot pasted it with members of the "TV Shows" category. Children seemed to relate the screenshot containing a person drawing more to other screenshots of live action videos than to screenshots of different arts and crafts from both live action and animated videos. This confirms the findings from the closed card sorting that the category "Make and Do" is not very clear for children, so the category was eliminated.

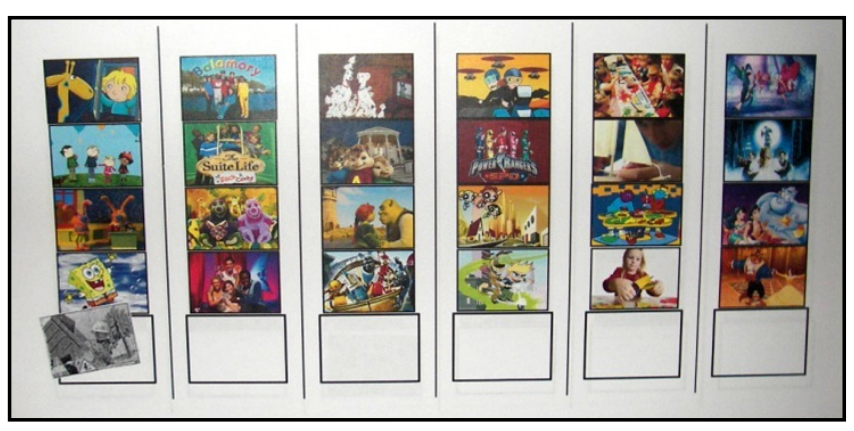

Figure 5. Match-to-sample completed by a Participant

Another result from the closed card sorting confirmed with the match-to-sample was the fact that the "Superheroes" category is very well understood. The superhero screenshot was pasted with screenshots from the "Superheroes" category by five out of six children $(p=0.17)$. And the one child that did not choose the superheroes group pasted it with screenshots from "Cartoons and Animations", category which the screenshot was also a member.

An interesting fact was that most children that related a low percentage of screenshots to the expected category during the closed card sorting, associated most or all screenshots to the ones from the category it is a best exemplar of during the match-to-sample task. And the opposite also occurred, children that inserted a high percentage of screenshots in the expected category during the closed card sorting pasted most screenshots in the match-to-sample activity with screenshots from a category it doesn't belong to. This could indicate that children's categorization abilities vary significantly, and while some participants could better categorize using category labels others could be best on matching a category member to others from the same group. For this reason it is recommended to conduct more than one type of card sorting activity while working with preschoolers, especially on studies with a small sample.

As children's abilities to categorize using different methods varies the type of categories they form and use could also differ, not just among the participants but also within children. Nguyen and Murphy (2003) concluded that preschoolers do not rely solely on one form of categorization. The authors examined script, taxonomic and evaluative categories for food items and stated that children can cross-classify items into multiple categories and use these categories for inductive inferences[10].

During the match-to-sample activity, we also noted that children are able to cross-classify screenshots based on videos' type (e.g., movies), format (e.g., cartoons) or genre (e.g., fairy tales) suggesting that they are not restricted to a single form of categorization. This indicates that in an EPG children could benefit from a significant overlap in categories rather than one replacing the other.

Therefore, for the prototype under development we decided to (1) make each category broader, so it could 
include all or most participants' grouping choices, and (2) overlap the categories.

We considered the screenshots pasted within each group of strong members of a category and evaluated how it could become a member of that specific category. This resulted in: "Cartoons and Animation", "TV Shows and Series", "Movies and Films", "Music and Songs", "Superheroes and Adventures", "Fairy Tales and Fantasy". In addition, the prototype will also include, as previous established with the closed card sorting task, categories related to television channels aimed at preschoolers. In the United Kingdom, where the prototype will be tested, they are: "Cbeebies", "Club House Disney", "Nick Jr" and "Cartoonito".

\section{CONCLUSIONS AND FUTURE WORK}

In conclusion, the closed card sorting can be used with preschool children and give some insight when designing for this age group. The method does have some constraints in comparing just two categories at a time but it can assist in finding when a category is understood.

The open card sorting demands more time. It could be an interesting technique to be used when young children are technology design partners but still needs to be further developed.

The match-to-sample was found really useful especially when combined with the closed card sorting; it can be used when children are informants in the design process contributing to the definition and refinement of categories that would best reflect children's choices.

The prototype under development incorporated the categories that emerged from this process. During the next stage of the research we will test in usability sessions the EPG and will analyze the impact card sorting tasks had on the way children look for specific videos members of one or several categories.

Further research has to be done on the impact these card sorting tasks could have in the design of menu entries and/or headings for different technology aimed at preschoolers.

\section{ACKNOWLEDGMENTS}

Our thanks to children and staff from: One World, Bright Start, Kipling Lions, Roundabout, Tarnerland and Wishing Tree Nurseries, Brighton. We are grateful to Renata Shimabukuru for her assistance with the study. And we also would like to thank Richard Castle and Myra Wiseman for their help with the statistics.

\section{REFERENCES}

1. Deak, G.O., Pick, A.D. and Ray, S.D. Matching and Naming Objects by Shape or Function: Age and Context Effects in Preschool Children. Developmental Psychology, 38 (4). 503-518.

2. Druin, A., Bederson, B.B., Boltman, A., Miura, A., Knotts-Callaham, D. and Plat, M. Children as our Technology Design Partners. in Druin, A. ed. The Design of Children's Technology,
Morgan Kaufmann Publishers, San Francisco, 1999, 51-72.

3. Goswami, U. Cognition in Children. Psychology Press Ltd, Hove, 1998.

4. Hanna, L., Risden, K., Czerwinski, M. and Alexander, K.J. The Role of Usability Research in Designing Children's Computer Products. in Druin, A. ed. The Design of Children's Technology, Morgan Kaufmann Publishers, San Francisco, 1999.

5. Hudson, W. Playing your cards right: getting the most from card sorting for navigation design, ACM, 2005, 56-58.

6. Kloo, D., Perner, J., Kerschhuber, A., Dabernig, S. and Aichhorn, M. Sorting between dimensions: Conditions of cognitive flexibility in preschoolers. Journal of Experimental Child Psychology, doi:10.1016 (j.jecp.2007.12.003).

7. Lyman, M. and Lewandowski, G. Genetic programming for association rules on card sorting data Proceedings of the 2005 conference on Genetic and evolutionary computation, ACM, Washington DC, USA, 2005.

8. Mervis, C.B. and Pani, J.R. Acquisition of Basic Object Categories. Cognitive Psychology, 12. 496-522.

9. Murray, G.C. and Reuter, K. Children's acquisition of categories and the implications for research in the development of classification schemes Annual Meeting of the American Society for Information Science \& Technology, 2005.

10. Nguyen, S.P. and Murphy, G.L. An Apple is More Than Just a fruit: Cross-Classification in Children's Concepts. Child Development, 74 (6). 1783-1806.

11. Nielsen, J. Card Sorting to Discover the Users' Model of the Information Space, 1995.

12. Scaife, M., Rogers, Y., Aldrich, F. and Davies, M. Designing for or designing with? Informant design for interactive learning environments Proceedings of the SIGCHI conference on Human factors in computing systems, ACM Press, Atlanta, Georgia, United States, 1997.

13. Smith, L. Emerging Ideas about Categories in Gershkoff-Stowe, L. and Rakison, D.H. eds. Building Object Categories in Developmental Time, Lawrence Erlbaum Associates, London, 2005, 159-170.

14. Sung, Y.-T., Chang, K.-E. and Lee, M.-D. Designing multimedia games for young children's taxonomic concept development Computers \& Education, 2008, 1037-1051. 


\section{APPENDIX A}

Triads of screenshots and the two categories presented for the closed card sorting.

\begin{tabular}{lll}
\hline Screenshots & Expected Category & Unexpected Category \\
\hline Ratatouille / Cars & Movies & Music \\
Bob the Builder / Dora the Explorer & Cartoons & Fairy Tales \\
Fraggle Rock / Tweenies & Music & Superheroes \\
Balamory / Tweenies (both make and do scenes) & Make and Do & Animals and Nature \\
Wild Show / Saving Species & Animals and Nature & Cartoons \\
Cinderella / Barbie Mariposa And Her Butterfly Fairy Friends & Fairy Tales & TV Shows \\
Ben 10 / Kimpossible & Superheroes & Around the World \\
Serious Amazon / George of the Jungle & Around the World & Cartoons \\
Teletubbies / Pocoyo & TV Shows & Superheroes \\
Balamory / Pingu & CBeebies (channel) & CITV (channel) \\
Goofy / Club House Disney & Disney (channel) & CBBC (channel) \\
\hline
\end{tabular}

\section{APPENDIX B}

Sets of screenshots handed to participants in each condition of the open card sorting task.

Condition 1

1. Wild Show, Save the Species, Barbie Mariposa And Her Butterfly Fairy Friends, Cinderella

2. Balamory, Balamory (make and do scene), Tweenies (music scene), Fraggle Rock

3. Goofy, Club House Disney, Pingu, Pocoyó

4. Ben 10, Dora the Explorer, Kimpossible, Powerpuff Girls

5. Cars, Bob the Builder, Ratatouille, Teletubbies

Condition 2

1. Wild Show, Save the Species, Cinderella, Balamory, Balamory (make and do scene)

2. Barbie Mariposa And Her Butterfly Fairy Friends, Tweenies (music scene), Fraggle Rock, Goofy, Club House Disney

3. Pocoyó, Pingu, Ben 10, Dora the Explorer, Powerpuff Girls

4. Kimpossible, Cars, Bob the Builder, Ratatouille, Teletubbies

\section{APPENDIX C}

Screenshots and the six categories presented for the match-to-sample task in each of the three conditions.

Condition 1

\begin{tabular}{|c|c|c|c|}
\hline Screenshots & Best Exemplar's Category & $\begin{array}{l}\text { Also Belongs to } \\
\text { Categories }\end{array}$ & $\begin{array}{l}\text { Not a Member of the } \\
\text { Categories }\end{array}$ \\
\hline Bob the Builder & Cartoons and Animations & - & $\begin{array}{l}\text { TV Shows, } \\
\text { Superheroes, Make and Do, } \\
\text { Fairy Tales }\end{array}$ \\
\hline Ratatouille & Movies & Cartoons and Animations & $\begin{array}{l}\text { Make and Do, Music, } \\
\text { Superheroes, Fairy Tales }\end{array}$ \\
\hline Ben 10 & Superheroes & Cartoons and Animations & $\begin{array}{l}\text { Make and Do, Movies, Fairy } \\
\text { Tales, Music }\end{array}$ \\
\hline
\end{tabular}


Condition 2

\begin{tabular}{|c|c|c|c|}
\hline Screenshots & Best Exemplar's Category & $\begin{array}{l}\text { Also Belongs to the } \\
\text { Categories }\end{array}$ & $\begin{array}{l}\text { Not a Member of the } \\
\text { Categories }\end{array}$ \\
\hline Teletubbies & TV Shows & - & $\begin{array}{l}\text { Cartoons and Animations, Fairy } \\
\text { Tales, Make and Do, Movies, } \\
\text { Superheroes }\end{array}$ \\
\hline $\begin{array}{l}\text { Balamory } \\
\text { (make and do scene) }\end{array}$ & Make and Do & TV Shows & $\begin{array}{l}\text { Superheroes, Movies, Fairy } \\
\text { Tales, Cartoons and Animations }\end{array}$ \\
\hline Cinderella & Fairy Tales & $\begin{array}{l}\text { Cartoons and Animations, } \\
\text { Movies }\end{array}$ & Superhero, Make and Do, Music \\
\hline \multicolumn{4}{|l|}{ Condition 3} \\
\hline Screenshots & Best Exemplar's Category & $\begin{array}{l}\text { Also Belongs } \\
\text { Categories }\end{array}$ & $\begin{array}{l}\text { Not a Member of the } \\
\text { Categories }\end{array}$ \\
\hline $\begin{array}{l}\text { Tweenies } \\
\text { (music scene) }\end{array}$ & Music & - & $\begin{array}{l}\text { Make and Do, Movies, Cartoons } \\
\text { and Animations, Superheroes, } \\
\text { Fairy Tales }\end{array}$ \\
\hline Balamory & TV Shows & - & $\begin{array}{l}\text { Cartoons and Animations, Fairy } \\
\text { Tales, Superheroes, Movies, } \\
\text { Make and Do }\end{array}$ \\
\hline Goofy & Cartoons and Animations & - & $\begin{array}{l}\text { Make and Do, Music, Movies, } \\
\text { Superheroes, Fairy Tales }\end{array}$ \\
\hline
\end{tabular}

